\title{
Developing a Framework for Potential Candidate Selection
}

\author{
Farzana Yasmin ${ }^{1}$, Mohammad Imtiaz Nur ${ }^{2}$, Mohammad Shamsul Arefin ${ }^{3}$ \\ Computer Science and Engineering, Chittagong University of Engineering and Technology \\ Chattogram, Bangladesh
}

\begin{abstract}
Recruitment is the process of hiring the right person for the right job. In the current competitive world, recruiting the right person from thousands of applicants is a tedious work. In addition, analyzing these huge numbers of applications manually might result into biased and erroneous output which may eventually cause problems for the companies. If these pools of resumes can be analyzed automatically and presented to the employers in a systematic way for choosing the appropriate person for their company, it may help the applicants and the employers as well. So in order to solve this need, we have developed a framework that takes the resume of the candidates, pull out information from them by recognizing the named entities using machine learning and score the applicants according to some predefined rules and employer requirements. Furthermore, employers can select the best suited candidates for their jobs from these scores by using skyline filtering.
\end{abstract}

Keywords-Information extraction; named entity recognition; machine learning; skyline queries

\section{INTRODUCTION}

Information extraction (IE) infers the process of automatically gisting of information in a strucutred way from unstructured and/or semi-structured machine-readable documents. The task involves the utilization of natural language processing (NLP). The present purpose of IE refers to the growing amount of information available in unstructured form [1].

Nowadays huge volume of documents are found online and offline. Extracting information from these vast volumes of data manually is time consuming. Moreover generating some pattern from the extracted information has recently been a new challenge and prime concern of the modern technological era.

Recruitment is the process of searching and selecting best candidates for filling the vacant positions of an organization. Recruitment process requires planning, requirements setup strategy, searching candidates, screening the candidates according to the requirements and evaluation of the candidates. These steps are usually conducted by the Human Resource (HR) department of any company. Whenever there is a job opening for the vacant positions, large amount of applications are dropped. On the other hand, the recruiters may search applicants from a job portal placing their requirements. In both cases, searching and screening the best candidates from these applicants after assessing the ablites and qualifications manually, takes huge amount of time, cost and effort of the HR department as the volume of data are big. If we can develop an efficient system for extracting information from the resumes and process these information in an automated way so that only the relevant applications are presented to the recruiters, it will ease the work of the HR management. An automated system for choosing the potential candidates that best suits the position's requirements can increase the efficiency of the HR agencies greatly.

Therefore, in order to make the recruitment process easy, effective and automated, we have developed a framework of potential candidate selection system. To perform this task we have chosen a domain of document information extraction which can be helpful in choosing the best potential candidates for any job openings i.e. CV/resume document. This development task involves the information extraction based on natural language processing i.e. tokenization, parsing, named entity recognizer (NER) and utilizes skyline query processing which works well in filtering the non-dominating objects from database and also makes a new addition to this domain.

So the objectives of the system development can be summerized as follows: 1) To design an efficient information extraction system from documents like curriculum vitae, 2) To generate scores on different features based on extracted information, 3) To perform appropriate filtering of information using skyline queries and 4) To generate proper ranking system for candidate selection.

The rest of the paper is presented as follows: In Section II related works of the candidate ranking system development has been portrayed. The system architecture and design is elaborated in Section III. Section IV represents the implementation of our work with some experimental results. And finally, a conclusion over the work has been drawn in Section V.

\section{RELATED WORK}

D. Celik [2] proposed an information extraction system for candidate selection where the information extraction was based on ontology. The proposed methodology used Ontology-based Resume Parser(ORP) to convert English and Turkish documents into ontological format. The proposed method constructed seven reference ontologies to extract the information and categorize them into one of these ontologies. Though the methodology worked good on information extraction but it did not describe any score generation mechanism to rank the candidates.

Another form of candidate selection was proposed by S. Kumari et. al. [3] where candidate selection was done by using 
Naïve Bayes algorithm for classifying the candidate profiles. They also considered employers importance criteria. No description given of how the information extraction are done. Also it requires GPRS connection every time as it is online based.

R. Farkas et al. [4] worked on a method of extracting information for career portal where the information of applicants' are stored in a uniform data structure named HRXML format. They used a CV parser to automatically extract data from the CV. It is basically template specific method and doesn't work for all formats of documents.

In [5], the authors used a hybrid cascade model for information extraction from CVs. In the first pass, the proposed method segments resume using Hidden Markov Model. The second pass uses HMM and SVM to extract further detailed information. The cascaded pipeline suffers from error propagation i.e. errors from first step are passed in the second pass and the precision and recall value decreases subsequently.

Information is extracted from resumes using basic techniques of NLP like word parsing, chunking, reg ex parser in [6]. Information like name, email, phone, address, education qualification and experience are extracted using pattern matching in this work. Some other online resume parsers are found in [7] \& [8].

An algorithm for CV information extraction is developed in [9] which works in two step. In the first step, raw texts are retrieved as resume blocks. Then in the next step they developed a mechanism to identify the fact information from the resume like named entities.

There also have been developed some works using skyline queries. [10], [11] \& [12] describes some algorithms for processing skyline queries with their implementation.

S. Patil et al. [13] developed a method for learning to rank resumes with the help of SVM rank algorithm. In [14], X. Yi et. al. applied a Structured Relevance Model to select resumes for a given post or to choose the best jobs for a given candidate based on their CV. In [15] job narration are transformed into queries and lookup in database is performed. The top-ranked candidates get selected automatically from these queries. Some authors exploit additional information like social media information along with information gained directly from resumes [16]. Moreover, [17] takes consideration of data collected from the LinkedIn profile and personality traits from the personal blogs of the candidates. In [18], digital resumes of candidates are generated by extracting data from social networking sites like Facebook, Twitter and LinkedIn. Candidates are evaluated based on their digital resume and ranked accordingly. In [19], CVs are filled in a predefined format and the scoring and ranking process is based on Analytic Hierarchy Process (AHP).

Though many works have been developed for candidate recruitment, the use of skyline query in this sceneraio is relatively new approach and we have implemented this novel approach in our framework.

\section{SySTEM ARCHITECTURE AND DESIGN}

The proposed framework works in 4 modules according to Fig. 1: Document processing module, Query Execution Module, Analysis \& Output module and Storage module.

\section{A. Processing Module}

1) $C V$ upload: First, the candidates may upload their resumes in the interface. After resumes are uploaded to the system it is considered as input for the processing module.Then information extraction process begins and we used a NLP module named spaCy [20] for the rest of the processing steps. Suppose, sample resumes as Fig. 2(a), (b) and (c) are uploaded in the system.

2) Conversion to text: The standard format of resumes for our system is considered english resumes in PDF format. At first, we need to convert the pdf into plain text using UTF-8 encoding. From [21], UTF stands for Unicode Transformation Format and '8' means it uses 8-bit blocks to represent a character. The number of blocks needed to represent a character varies from 1 to 4 . UTF-8 is a compromise character encoding that can be as compact as ASCII but can also contain any Unicode characters.

3) Tokenization: After conversion to text, now we have our necessary text file. We start reading the text file and tokenize the whole document. Tokenization is the process of splitting a document into its smallest meaningful pieces named tokens. Tokenization is done using the language rule i.e. removing the white space, checking the exception rules like punctuation checking, abbreviation rules, etc.

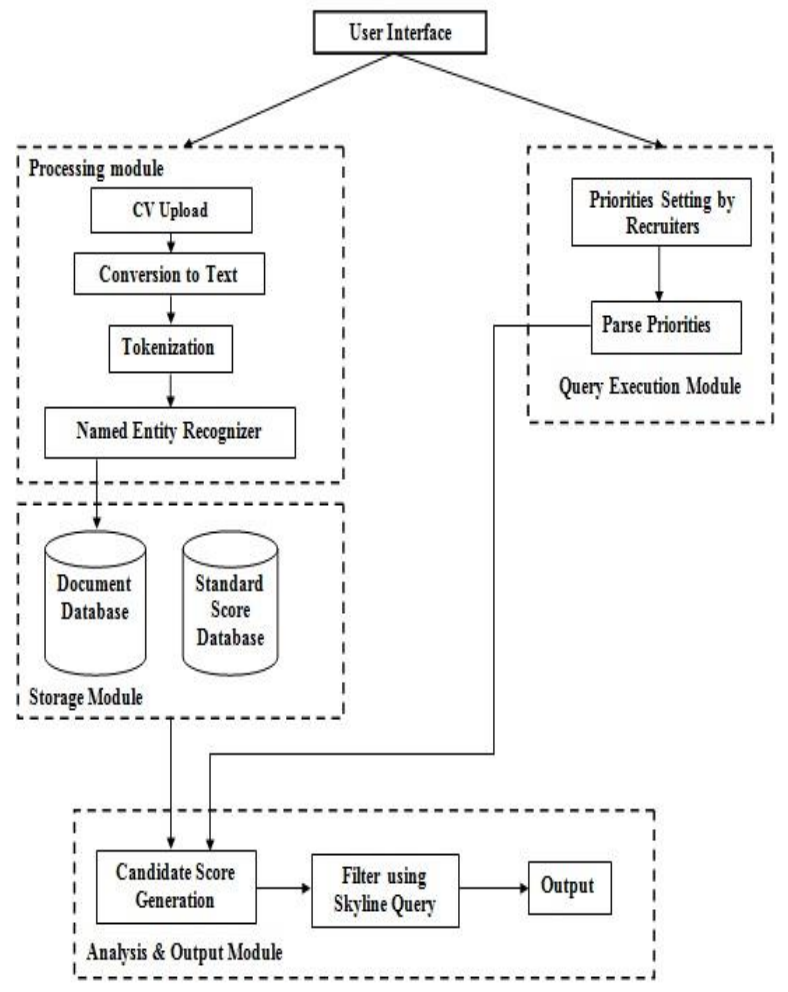

Fig. 1. System Architecture of Potential Candidate Selection System. 


\begin{tabular}{|c} 
Adam Wang (Male) \\
XXXX Company of Bejing, \\
Beijing City, 100007 \\
1364-110-XXX \\
wangXXX@hotmail.com \\
Education Background \\
From Sept. 2000 to Apr. 2003, I got master degree \\
rom University of XXX in computer software \\
engineering. \\
From Sept. 1996 to July. 2000, I got bachelor degree \\
from School of XXX and major in computer science \\
and technology. \\
Experience \\
From Mareh 2003 to now, working on Human Face \\
Recognition System in XXXX Company of Beijing \\
From June 2001 to March 2003, working on \\
Content-Based Intelligent Image Retrieval System in \\
Research Center of XXX Company \\
From Sept. 2000 to May 2001, wotking on \\
Intelligent Highway Distress Detection System in \\
National Lab. Of XXX University \\
Interests \\
Reading, music, and jogging \\
\hline
\end{tabular}

(a)

$\mathrm{ABC}$

Cell: +8801680671851

E-mail: abc@gmail.com

RESEARCH INTEREST

Data Mining, Artificial Intelligence, Machine

Learning, Algorithm Design, Data Structure.

EDUCATIONAL QUALIFICATIONS

\# B.Sc. in CSE- October 2015

CUET, Bangladesh.

Result: CGPA 3.81 (out of 4.00)

Class Position: $2^{\text {nd }}$ out of 113 students

WORK EXPERIENCES

\# Chittagong University of Engineering \&

Technology, Chittagong-4349, Bangladesh

> Joined as "Lecturer" as on 2016

Duration : 2016- Present

(b)

\section{Ishraq Rayeed Ahmed}

+8801717342569, ishraqrayeed@gmail.com, www.irahmed.wordpress.com

Research Interests

Pavement Materials and Design, Urban and Public Transportation System, Traffic Emissions and Air Quality, Transportation Safety, Intelligent Transportation System Education

Bachelor of Science, Civil Engineering, March 2016 BUET at Dhaka, Bangladesh

CGPA: 3.24/4.00

Technical Skills

Scientific Computing \& Simulation Tools: MATLAB, R Project, ArcGIS, VisSim, EPAnet

Structural Design Software: AutoCAD, SAP, ETABS, GRASP

Programming Languages: $\mathrm{C}++$, Python

Graphics Design Software: Adobe Photoshop, Adobe Illustrator, 3D Studio Max

\section{Publications}

- Ahmed, I.R; Mondal, A.R; Noor, A.U, “Assessment Of Pedestrian Perception Towards

Pedestrian Crossing Facilities In Dhaka Metropolitan City: A Study Based On Observation and Survey”, (IICSD-2015), DUET, Dhaka.

(c)

Fig. 2. (a), (b) and (c) Sample Resumes.
4) Named entity recognition: Named entity recognition (NER) is the most important task to do next. The success of the extraction process mainly depends on the accurately recognized entities from a resume. The subtask of information extraction that seeks to locate and classify named entity mentions in unstructured text into pre-defined categories such as the person names, organizations, email, phone, address, time, quantities, numeric values, etc. can be defined as Named entity recognition [22]. A statistical model is used to classify our desired entities in a standard resume like name, date of birth, email, phone number, university, education, major, publications, experience, skills, etc. The NER training model is designed using incremental parsing and residual CNNs. In case of training our model (Fig. 3) with the desired annotation we used resumes in JSON format.

The adapted algorithm of spaCy's NER training module is provided below:

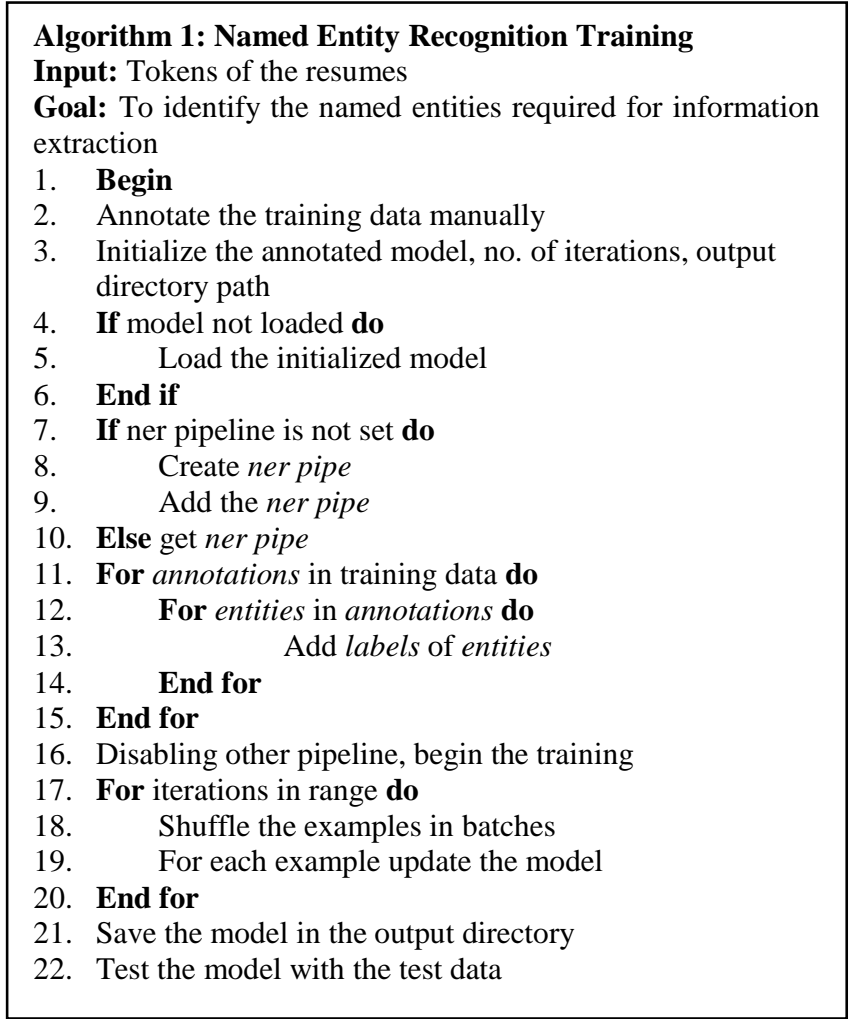

At first, we have to manually annotate our training data in JSON format (2). Then we load or build the NER model (step 4-6). For training the NER model with our custom entities, now we add the labels for each annotations (step 11-15). For starting the training of our NER model, we must disable other pipeline components like tokenizer, tagger of spaCy (step 16). Then we shuffle and loop over our training examples (step 18). At each word the model makes a prediction. It then consults the annotations to see whether it was right. If it was wrong, it makes adjustment of the weight so that the correct action will score higher next time (step 19). Then we save the model (step 21) and test it to make sure the entities in the test data are recognized correctly (step 22). 


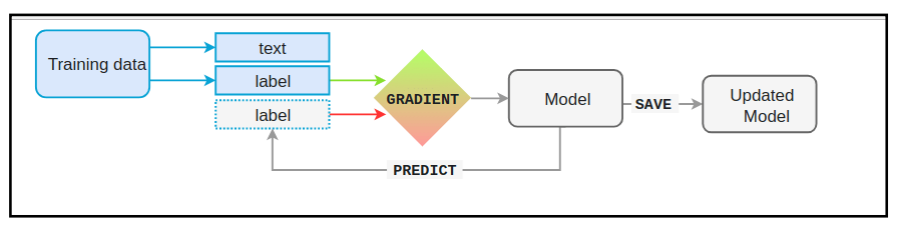

Fig. 3. SpaCy's NER Model Training Process (Source: [23]).

After the validation of the training of the NER model, now we use this model to extract the values of the entities from the resumes as blocks. The recognized entity values are stored block-wise in a row of a table for each candidate in the storage module. If we send the resumes in the NER model, the table of the extracted information takes the form like Table I, Table II, Table III, Table IV and Table V.

\section{B. Storage Module}

Storage module stores information processed by the processing module. The extracted information table after the entites are recognized are stored in the document database. We set the scores for different criterias based on some predefined hypothetical rules. The total storage is required for the candidate score generation in the analysis and output generation phase. The rules and scores that are set as lookup tables (Tables VI and VII) in the score database are given.

\begin{tabular}{|c|}
\hline $\begin{array}{c}\text { Rule for Experience: } \\
\text { If }(\text { Designation }==\text { Required experience designation }) \\
\text { If }(\text { Experience }==\text { Required position experience }) \\
\text { Score }[\text { Experience }]=5 \\
\text { Else } \quad \text { If }(\text { Total Experience } \geq \text { Required experience }) \\
\text { Score }[\text { Experience }]=2 \\
\text { Else } \text { Score }[\text { Experience }]=0\}\end{array}$ \\
\hline $\begin{array}{l}\text { Rule for Skills: } \\
\text { If (Candidate_skill== Required skill) } \\
\text { Score }[\text { skilll] =Score[skill] } 1 \\
\text { Else } \text { Score }[\text { skill }]=0\end{array}$ \\
\hline Rule for Certification: \\
\hline $\begin{array}{l}\text { If }(\text { Candidate_certification }==\text { Required certification }) \\
\text { Else } \quad \begin{array}{l}\text { Score }[\text { Certification] }=\text { Score }[\text { Certification }]+1 \\
\text { Score }[\text { Certification] }=0\end{array}\end{array}$ \\
\hline
\end{tabular}

TABLE. I. EXTRACTED INFORMATION OF PERSONAL INFORMATION BLOCK

\begin{tabular}{|l|l|l|l|}
\hline Name & Phone & Email & Date of Birth \\
\hline Farzana Yasmin & 01680671851 & farzanaefu@gmail.com & 30 Sep 1993 \\
\hline Mohammad Imtiaz Nur & 01818772617 & imti.nur@gmail.com & $07-09-1992$ \\
\hline Ohidul Islam & 01617224955 & ohid@gmail.com & $14-12-1989$ \\
\hline Faisal Karim & 01826564578 & faisal90@ outlook.com & 13.05 .1994 \\
\hline Md. Intishar ur & 01747678878 & intishar788@gmail.com & $6 / 1 / 1983$ \\
\hline Ananna Das & 01918793180 & anannadas@yahoo.com & $5-23-1987$ \\
\hline Hasibul Haq & 01922935210 & h.haq602@yahoo.com & 3 April 1990 \\
\hline
\end{tabular}

TABLE. II. EXTRACTED INFORMATION OF EDUCATIONAL INFORMATION BLOCK

\begin{tabular}{|l|l|l|l|l|}
\hline Name & Degree & Major & Institution \\
\hline Farzana Yasmin & B.Sc & CSE & $\begin{array}{l}\text { Chittagong University of } \\
\text { Engineering \& Technology }\end{array}$ \\
\hline Mohammad Imtiaz Nur & Bachelor in Science & CSE & CUET & 3.81 \\
\hline Ohidul Islam & B.Sc., M.Sc. & Electrical Engineering & BUET, Jahangirnagar University & $3.72,3.96$ \\
\hline Faisal Karim & B.Sc., PhD & CSE & $\begin{array}{l}\text { Chittagong University, University } \\
\text { of Houston }\end{array}$ & 3.50 \\
\hline Md. Intishar Nur & Bachelor in Science & Computer Science & IUT & 3.46 \\
\hline Ananna Das & LLB & Law & Premier University \\
\hline Hasibul Haq & Bachelor in Business Administration & Finance & IIUC \\
\hline
\end{tabular}

TABLE. III. EXTRACTED INFORMATION OF PUBLICATION BLOCK

\begin{tabular}{|l|l|}
\hline Name & Publication \\
\hline Farzana Yasmin & International Journal, International Conference \\
\hline Mohammad Imtiaz Nur & -- \\
\hline Ohidul Islam & International Conference \\
\hline Faisal Karim & International Journal, International Journal, International Journal, International Conference \\
\hline Md. Intishar Nur & -- \\
\hline Ananna Das & -- \\
\hline Hasibul Haq & -- \\
\hline
\end{tabular}


TABLE. IV. EXTRACTED INFORMATION OF EXPERIENCE BLOCK

\begin{tabular}{|l|l|l|l|}
\hline Name & Company Worked at & Experience & Designation \\
\hline Farzana Yasmin & CUET & 3 yrs & Lecturer \\
\hline Mohammad Imtiaz Nur & SAPL & 2 yrs & Executive Programmer \\
\hline Ohidul Islam & PDB & 7 yrs & Executive Engineer \\
\hline Faisal Karim & MatWorks & 2.5 yrs & Senior Programmer \\
\hline Md. Intishar Nur & BSRM & 6 months & Assistant Programmer \\
\hline Ananna Das & -- & 0 & -- \\
\hline Hasibul Haq & Dhaka Bank Ltd. & 5 yrs & Senior Officer \\
\hline
\end{tabular}

TABLE. V. EXTRACTED INFORMATION OF OTHERS BLOCK

\begin{tabular}{|l|l|l|}
\hline Name & Skills & Certification \\
\hline Farzana Yasmin & C, C++, PHP, Python, HTML, Java Script & CCNA \\
\hline Mohammad Imtiaz Nur & Angualar, JavaScript, PHP, Java & Mobile Apps Training \\
\hline Ohidul Islam & Python, Java, Javascript, Rubi on rails & -- \\
\hline Faisal Karim & C\#, C++, PHP, Html, CSS, Javascript & -- \\
\hline Md. Intishar Nur & Matlab, C++ & -- \\
\hline Ananna Das & MS Word, MS Office & -- \\
\hline Hasibul Haq & MS Word, MS Office, Linux & - \\
\hline
\end{tabular}

TABLE. VI. SCORE LOOKUP TABLE (PUBLICATION)

\begin{tabular}{|c|c|c|c|c|c|c|c|c|c|}
\hline \multicolumn{6}{|c|}{ International Journal } & \multicolumn{4}{|l|}{ Conference } \\
\hline Indexing & Score & Publisher & Score & Impact Factor & Total & $\begin{array}{l}\text { International } \\
\text { Conference }\end{array}$ & Score & Others & Score \\
\hline SCI & 1 & Nature & 1 & \multirow{5}{*}{ Value } & \multirow{5}{*}{$\begin{array}{l}\text { Sum of } \\
\text { Indexing, } \\
\text { Publisher and } \\
\text { Impact Factor } \\
\text { score }\end{array}$} & \multirow{5}{*}{$\begin{array}{l}\text { Match the keyword } \\
\text { 'International } \\
\text { Conference' }\end{array}$} & \multirow{5}{*}{.2} & \multirow{5}{*}{$\begin{array}{l}\text { If doesn't satisfy } \\
\text { other criteria of } \\
\text { publication }\end{array}$} & \multirow{5}{*}{.1} \\
\hline SCIE & .75 & $\begin{array}{l}\text { Springer, } \\
\text { IEEE, Wiley, } \\
\text { Elsevier }\end{array}$ & .5 & & & & & & \\
\hline SCOPUS & .5 & \multirow{3}{*}{ Others } & \multirow{3}{*}{.1} & & & & & & \\
\hline Others & .4 & & & & & & & & \\
\hline Predatory & 0 & & & & & & & & \\
\hline
\end{tabular}

TABLE. VII. SCORE LOOKUP TABLE (EDUCATION)

\begin{tabular}{|c|c|c|c|c|c|c|c|}
\hline Inst. Ranking & PhD Score & M.Sc. Score & B.Sc. Score & $C G P A$ & & Major & \\
\hline $1-200$ & 10 & 5 & 2 & Rule & Score & Rule & Score \\
\hline $201-500$ & 9.5 & 4.8 & 1.9 & $\geq 3.75$ & 4 & \multirow{9}{*}{$\begin{array}{l}\text { Keyword Matching } \\
\text { with Requirement }\end{array}$} & \multirow{9}{*}{2} \\
\hline $501-1000$ & 9 & 4.6 & 1.8 & $3.5-3.74$ & 3 & & \\
\hline $1001-1500$ & 8.5 & 4.4 & 1.7 & $3.0-3.49$ & 2 & & \\
\hline $1501-2000$ & 8 & 4.2 & 1.6 & $2.5-2.99$ & 1 & & \\
\hline $2001-2500$ & 7.5 & 4 & 1.5 & Others & 0 & & \\
\hline $2501-3000$ & 7 & 3.8 & 1.4 & & & & \\
\hline $3001-3500$ & 6.5 & 3.6 & 1.3 & & & & \\
\hline $3501-4000$ & 6 & 3.4 & 1.2 & & & & \\
\hline Others & 5.5 & 3.2 & 1.1 & & & & \\
\hline
\end{tabular}

TABLE. VIII. REQUIREMENT SETTING TABLE

\begin{tabular}{|l|l|}
\hline Job_criteria & Keywords \\
\hline Skills & C++, Java, PHP \\
\hline Experience & $0-3$ yrs as Executive/ Senior Programmer \\
\hline Major & CSE, EEE \\
\hline
\end{tabular}




\section{Query Execution Module}

1) Priorities setting by the recruiter: In the UI, employers set the requirements for the vacant positions of their company. For example, for Senior Programmer position, the employer sets the following requirements as Table VIII for each criteria.

2) Parse priorities: The system will then parse these requirements of the employer in the query execution module.

\section{Anaysis and Output Module}

1) Candidate Score Generation: After parsing the requirement of the employer, the system will start the score table generation of each candidate according to the employer priority and previously set standard score for different categories from the score database. The algorithm of candidate score generation is given below:

\begin{tabular}{|c|c|}
\hline \multicolumn{2}{|r|}{$\begin{array}{l}\text { Algorithm 2: Candidate Score Generation } \\
\text { Input: Extracted information stored in Excel file } \\
\text { Goal: To generate score of each candidate in each criterion }\end{array}$} \\
\hline 1. & Begin \\
\hline 2. & Initialize Scores object with unique job_criteria \\
\hline 3. & Initialize an empty Score_table list \\
\hline & For each row in excel do \\
\hline 5. & Set Scores object value to zero \\
\hline 6. & For each job_info details do \\
\hline 7. & Find(Excel(column)) \\
\hline 8. & If job_criteria $==$ Excel $($ column $)$ do \\
\hline 9. & If keyword matches with column value do \\
\hline 10. & $\begin{array}{l}\text { Calculate the Scores value as: } \\
\text { Scores [job_criteria] }+=\text { score set for the } \\
\text { criteria in the score database }\end{array}$ \\
\hline 11. & Else skip \\
\hline 12. & Else skip \\
\hline 13. & End For \\
\hline 14. & Push Scores values in Score_table \\
\hline 15. & End for \\
\hline 16. & Set the mandatory required job_criteria \\
\hline 17. & If Scores [mandatory_job_criteria] $=0$ do \\
\hline 18. & Delete the score row from the Score table \\
\hline
\end{tabular}

The extracted information stored in the lookup table in document database is retrieved (step 7-8) and matched with the keywords stored in the job_info_details table (step 9). If match found, the corresponding values are calculated as the rules set in the standard score table (step 10).

If multiple keywords are matched for a specific criteria, then they are stored as aggregated sum. For example, if multiple skills match, then all the skill values are added and stored in the skill column for that candidate.

For education score generation, the degrees and the ranking of the institutions they are acquired from are checked and scores for the institution and degree is put on the score table.

For the publication column, internation conference, international journal keywords are searched and matched. If found, the number of occurences are counted. The lists of SCI, SCIE, SCOPUS and predatory journals are sotred in the database. The journal names are matched with these list. If match found, scores are calculated accordingly.

For the experience column, at first it is checked that the candidate is fulfilling the requirements for the given job. If yes, the experience score is calculated. If relevant experience is not fulfilled according to the requirements, the total experience is checked and given a score.

For skills an certifications score calculation, the requirement of the employers are checked. If matched, the information is given a value for each matched keywords.

If any column information contains missing value, then they are considered as zero in the score calculation. The calculated score is stored in that specific criteria column of the score table. After being scored in each criteria, now a table is generated which is score of each candidate (step 14).

The sample score table of the resumes of Table I are depicted below in Table IX.

TABLE. IX. SAMPLE SCORE TABLE

\begin{tabular}{|l|l|l|l|l|l|l|l|}
\hline $\begin{array}{l}\text { CV } \\
\text { no. }\end{array}$ & Degree & $\begin{array}{l}\text { Publicati } \\
\text { on }\end{array}$ & CGPA & Skills & Experience & Major & Total \\
\hline 1 & 1.1 & .2 & 4 & 1 & 2 & 2 & 10.3 \\
\hline 2 & 1.1 & 0 & 2 & 2 & 5 & 2 & 12.1 \\
\hline 3 & 5 & .1 & 8 & 1 & 0 & 2 & 16.1 \\
\hline 4 & 10.1 & 54.2 & 3 & 2 & 5 & 2 & 76.3 \\
\hline 5 & 1.1 & 0 & 2 & 0 & 2 & 2 & 7.1 \\
\hline 6 & 0 & 0 & 2 & 0 & 2 & 0 & 4 \\
\hline 7 & 0 & 0 & 1 & 0 & 0 & 0 & 1 \\
\hline
\end{tabular}

The first candidate had a B.Sc degree from Chittagong University of Engineering \& Tech. And its ranking goes to institution category others. So the value for degree from the lookup Table VI is 1.1. She also has an international conference publication so the score is .2. The first candidate had the matching skill $\mathrm{C}++$, experience of 3 years but as lecturer and major CSE. So the first candidate get scores according to the rules.

The scores of the other candidates will be calculated as the 1st candidate. The degree of 6th and 7th candidate doesn't match the required degree database as we have only condidered technical degrees and so the missing value is scored as zero. Accordingly, the 5th, 6th and 7th candidates doesn't match the skills requirement and so they get a zero in skills field. Now if we select any field as mandatory, the row containing zero in that field will be deleted.

2) Filter using skyline query: A skyline is defined as those points in a dataset those cannot be worse than any other point. A point dominates other points if it is as greater or equal in all criteria and greater in at least one criterion. A study in [24] states that during the past two decades, skyline queries are applied in several multi-criteria decision support problems. Skyline query utilizes the idea of skyline operator. There are several algorithms for the implementation of skyline operator like using directly in SQL queries, block nested loop, divide 
and conquer $(\mathrm{D} \& \mathrm{C})$, branch and bound, map reduce etc. We have used the block nested loop (BNL) and D\&C method. Applying skyline queries on the score table according to employers' priorities, now the dominant applicants will be filtered. We can explain the working procedure of skyline query using Table IX. According to BNL, we compare all the data points with all other points. We keep the points that can dominate other points in all criteria and at least in one dimension. The points dominated are discarded from the list. Those points are considered to be skyline that dominates others or maybe a part of the skyline if they neither dominates nor dominated by others. For performing skyline filtering, the categories are to be selected by the recruiters. As the employer placed the requirements for only skills, major and experience category, so comparing the data points of these categories of Table IX we find that Candidate 2 and 4 dominates the other candidates in the required criteria as they contain either equal or higher value in every criteria than the other five. After applying skyline we get that candidate $2 \& 4$ best suits for the job and others are discarded from the list as depicted in Table X.

According to $\mathrm{D} \& \mathrm{C}$, first we will divide the list into $\mathrm{m}$ partitions recursively. Then a local skyline is calculated for each partition. Then we merge these local skylines for calculating the global skyline. And finally these global skylines are the best candidates for the job.

3) Output generation: The system output will show the result of the potential candidates after the filtering process. The output will be sorted according to the score obtained and personal details like name, email, phone number of each candidate will be displayed. The sample output generation is shown in Table XI.

The algorithm is depicted below:

\begin{tabular}{|c|c|}
\hline \multicolumn{2}{|r|}{$\begin{array}{l}\text { Algorithm 3: Filtering Using Skyline Query (BNL method) } \\
\text { Input: Generated Score_list, candidate (1), candidate (2),... } \\
\text { candidate(n) } \\
\text { Goal: To filter the total candidate, create the best candidates list and } \\
\text { remove the non dominant candidates }\end{array}$} \\
\hline 1. & Begin \\
\hline 2. & Initialize an empty best_candidates list \\
\hline 3. & Set flag 'passed'= true for all Candidates \\
\hline & For i to $\mathrm{n}$ of score_list \\
\hline 5. & If $($ passed $==$ false $\|$ candidate $(\mathrm{i})==$ candidate $(\mathrm{n}))$ \\
\hline 6. & Continue \\
\hline 7. & $\begin{array}{l}\text { Set } \text { compare_list }=\text { score_table filtered by } \text { (passed=true } \\
\& \& \text { candidate }(\mathrm{i})\end{array}$ \\
\hline 8. & For each job_criteria do \\
\hline 9. & If $($ candidate $(\mathrm{i}) .[$ criteria $] \geq$ candidate $(\mathrm{i}+1)$. [criteria] $)$ \\
\hline 10. & Candidate $(i+1) \cdot$ passed $=$ false \\
\hline 11. & $\begin{array}{l}\text { Else if (candidate (i). [criteria] < candidate } \\
(\mathrm{i}+1) \text {. [criteria]) }\end{array}$ \\
\hline 12. & $\{$ Candidate (i) $\cdot$ passed $=$ false \\
\hline 13. & Break\} \\
\hline 14. & End If \\
\hline 15. & End for \\
\hline & Set best_candidates list $=$ candidates with $($ passed $=$ true $)$ \\
\hline & End for \\
\hline
\end{tabular}

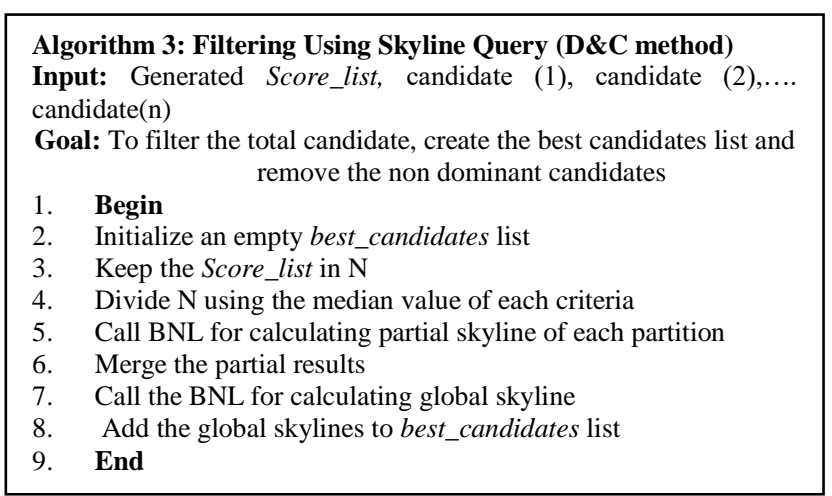

TABLE. X. SCORE TABLE AFTER FILTERING USING SKYLINE QUERY

\begin{tabular}{|l|l|l|l|}
\hline CV no. & Skills & Experience & Major \\
\hline 2 & 2 & 5 & 2 \\
\hline 4 & 2 & 5 & 2 \\
\hline
\end{tabular}

TABLE. XI. OUTPUT GENERATION

\begin{tabular}{|l|l|l|l|l|l|l|}
\hline $\begin{array}{l}\text { CV } \\
\text { No. }\end{array}$ & Name & Phone & Email & $\begin{array}{l}\text { Skil } \\
\text { ls }\end{array}$ & $\begin{array}{l}\text { Experie } \\
\text { nce }\end{array}$ & $\begin{array}{l}\text { Maj } \\
\text { or }\end{array}$ \\
\hline 2 & $\begin{array}{l}\text { Mohammad } \\
\text { Imtiaz Nur }\end{array}$ & $\begin{array}{l}0181877 \\
2617\end{array}$ & $\begin{array}{l}\text { imti.nur@ } \\
\text { gmail.com }\end{array}$ & 2 & 5 & 2 \\
\hline 4 & Faisal Karim & $\begin{array}{l}0182656 \\
4578\end{array}$ & $\begin{array}{l}\text { faisal90@ } \\
\text { outlook.co } \\
\text { m }\end{array}$ & 2 & 5 & 2 \\
\hline
\end{tabular}

\section{IMPLEMENTATIONS AND EXPERIMENTS}

In this section, we have described the implementation and experimental setup of our system with necessary illustrations.

\section{A. Experimental Setup}

Potential candidate selection system has been developed on a machine having Windows $10,2.50 \mathrm{GHz}$ Core i5-3210 processor with 12GB RAM. The system has been developed in Python 3.7.3, Asp.Net Core and Angular5 in the front end and MS SQL Server is used in the back end for storing related data to complete this project.

\section{B. Implementation}

At the beginning of our system workflow, resume documents are fed into the system. All the resumes are stored in a file according to the specific job id. These resumes are then converted into text format using UTF-8 encoding and stored in a file named lookup.py.

Once we have found the extracted information table, it is stored in the document database.

On the other hand, employers set the necessary information for setting the requirements of each criteria. Job_info_details table holds the columns like Job_info ID, Keyword, Job Criteria Name i.e. the information set by the recruiters on the requirements setting step. For the specific job position, extracted information table can be uploaded next for score generation.

After scoring according to the rules set, the system generates the score table. This table can be downloaded by the 
TABLE. XVI. RESPONSE TIME OF SKYLINE FILTERING

\begin{tabular}{|c|c|c|c|c|c|c|}
\hline \multirow{3}{*}{$\begin{array}{l}\text { No of } \\
\text { Resume }\end{array}$} & \multicolumn{6}{|c|}{ Response time (msec) } \\
\hline & \multicolumn{2}{|c|}{5 criteria } & \multicolumn{2}{|c|}{7 criteria } & \multicolumn{2}{|c|}{9 criteria } \\
\hline & BNL & $\mathrm{D} \& \mathrm{C}$ & BNL & $D \& C$ & BNL & D\&C \\
\hline 10 & 3.09 & 3.08 & 6.24 & 3.98 & 15.41 & 6.55 \\
\hline 50 & 13.01 & 3.73 & 24.00 & 5.61 & 32.69 & 10.17 \\
\hline 100 & 13.49 & 4.10 & 24.08 & 7.66 & 40.85 & 19.26 \\
\hline 200 & 17.33 & 4.98 & 24.466 & 7.77 & 48.445 & 22.90 \\
\hline
\end{tabular}

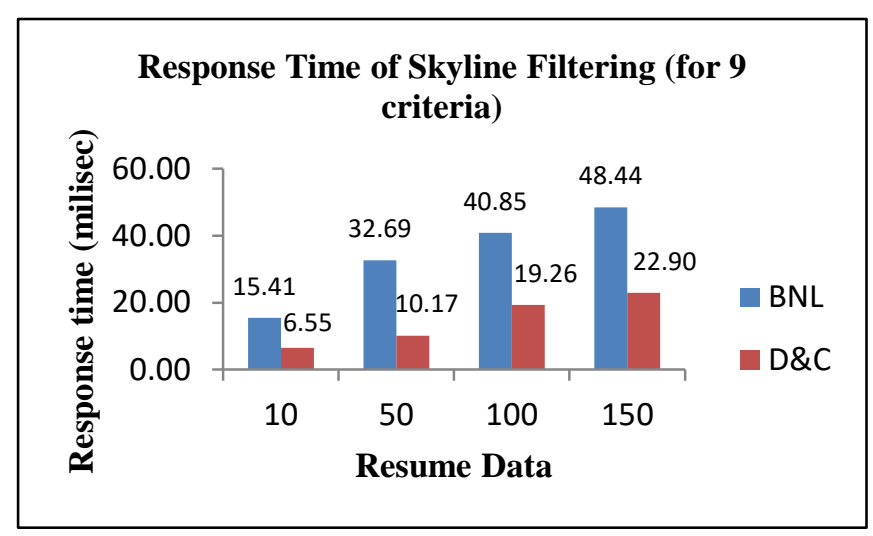

Fig. 5. Response Time of BNL \& D and C for 9 Criteria.

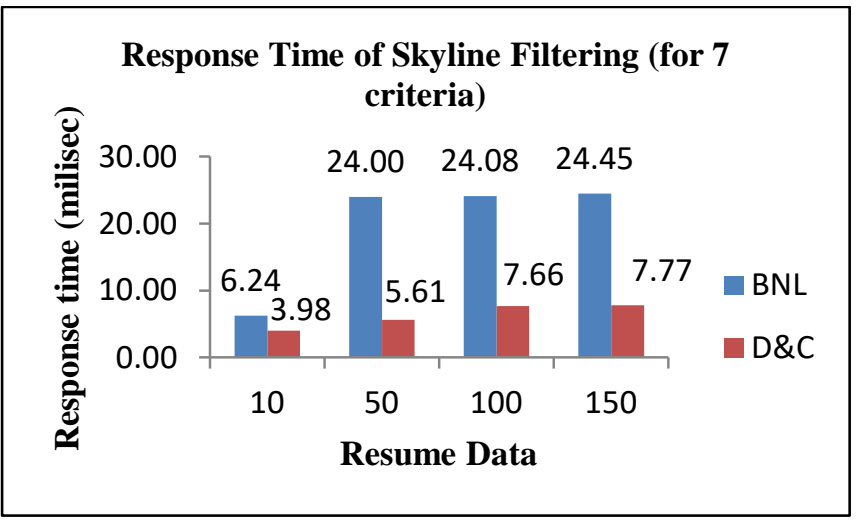

Fig. 6. Response Time of BNL and D and C for 7 Criteria.

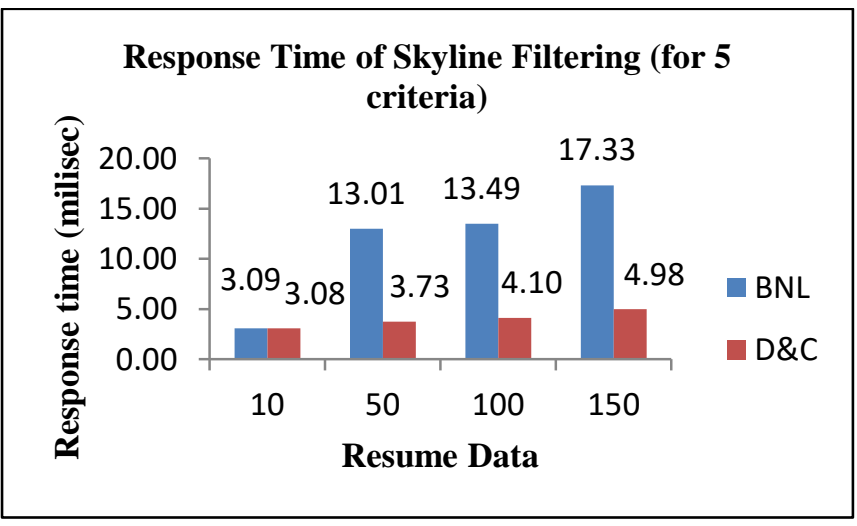

Fig. 7. Response Time of BNL and D and C for 5 Criteria.

\section{CONCLUSION}

In this paper, we have narrated the idea of a candidate selection system which finds the best potential candidates by extracting information and filtering using skyline query. Automating the total task may help the HR agencies by reducing time, cost and effort of searching and screening the pioneer applicants from vast applications. There are many automated candidate ranking system available online. But we have developed a novel idea of using skyline query in filtering and returning the dominant candidates for the job specified. Skyline queries are mostly applied in multidimensional decision application. In candidate filtering, the implementation of skyline is new and we have applied this novel approach in an efficient manner. In the system performance evaluation, we have used 150 resumes of technical background in testing of the system and found that, the system works in an efficient way of returning best candidates by matching the given requirements with qualifications of the candidates. Altogether the system performs better in filtering the documents as well as the candidates based on the information extracted from the resume documents. We have also compared the performance of two skyline filtering method and found out that D\&C method returns faster response than BNL method. Our system works for only English documents currently. In future, we hope to extend it for Bangla resumes as it is fifth most spoken native language in the world by incorporating Bangla Language Processing and test the system performance accordingly.

\section{REFERENCES}

[1] Information Extraction, https://en.wikipedia.org/wiki/Information_ extraction.

[2] Celik, D., "Towards a semantic-based information extraction system for matching resumes to job openings," Turkish Journal of Electrical Engineering \& Computer Sciences. vol. 24, pp. 141-159 (2016).

[3] Kumari, S., Giri, P., Choudhury, S., Patil, S.R., "Automated resume extraction and candidate selection system," In: International Journal of Research in Engineering and Technology, e-ISSN. 2319-1163, p-ISSN. 2321-7308, vol. 03, issue. 01 (2014).

[4] Farkas, R., Dobó, A., Kurai, Z., Miklós, I., Nagy, Á., Vincze, V., Zsibrita, J., "Information extraction from hungarian, english and german cvs for a career portal," In: Prasath R., O'Reilly P., Kathirvalavakumar T. (eds) Mining Intelligence and Knowledge Exploration. Lecture Notes in Computer Science, vol. 8891, Springer, Cham (2014).

[5] K. Yu, G. Guan, M. Zhou, "Resume information extraction with cascaded hybrid model," In Proceedings of the $43^{\text {rd }}$ Annual Meeting of the Association for Computational Linguistics, pp. 499-506, Ann Arbor, June 2005.

[6] Information Extraction from CV, https://medium.com/@divalicious. priya/information-extraction-from-cv-acec216c3f48.

[7] Writing Your Own Resume Parser, https://www.omkarpathak.in/ 2018/12/18/writing-your-own-resume-parser/.

[8] Resume Parser, https://github.com/bjherger/ResumeParser.

[9] Chen, J., Zhang, C., Niu, Z., "A two-step resume information extraction algorithm," Mathematical Problems in Engineering, vol. 2018, Article ID 5761287 (2018).

[10] Shah, S., Thakkar, A., Rami, S., "A survey paper on skyline query using recommendation system," In: International Journal of Data Mining And Emerging Technologies, vol. 6, issue. 1, pp. 1-6, ISSN. 2249-3212 (2016).

[11] Kalyvas, C., Tzouramanis, T., "A survey of skyline query processing," 2017. 
[12] Papadias, D., Tao, Y., Fu, G., Seeger, B., "An optimal and progressive algorithm for skyline queries," In: ACM SIGMOD International Conference on Management of Data, pp. 467-478 (2003).

[13] Patil, S., Palshikar, G.K., Srivastava, R., Das, I., "Learning to rank resumes," In: FIRE, ISI Kolkata, India (2012).

[14] Yi, X., Allan, J., Croft, W.B., "Matching resumes and jobs based on relevance models" In: SIGIR, Amsterdam, The Netherlands, pp. 809810 (2007).

[15] Rode, H., Colen, R., Zavrel, J., "Semantic CV search using vacancies as queries," In: 12th Dutch-Belgian Information Retrieval Workshop, Ghent, Belgium, pp. 87-88 (2012).

[16] Bollinger, J., Hardtke, D., Martin, B., "Using social data for resume job matching," In: DUBMMSM, Maui, Hawaii, pp. 27-30 (2012).

[17] Faliagka, E., Ramantas, K., Tsakalidis, A., Tzimas, G., "Application of machine learning algorithms to an online recruitment system," In: Seventh International Conference on Internet and Web Applications and Services, Stuttgart, Germany, pp. 215-220 (2012).
[18] Dandwani, V., Wadhwani, V., Chawla, R., Sachdev, N., Arthi, C.I., "Candidate ranking and evaluation system based on digital footprints," In: IOSR Journal of Computer Engineering (IOSR-JCE), e-ISSN. 22780661, p-ISSN. 2278-8727, vol. 19, issue. 1, ver. 4, pp. 35-38 (2017).

[19] Faliagka, E., Ramantas, K., Tsakalidis, A., Viennas, M., "An integrated e-recruitment system for cv rankıng based on ahp," In: 7th International Conference on Web Information Systems and Technologies, Noordwijkerhout, The Netherlands, (2011).

[20] SpaCy, https://spacy.io/.

[21] UTF-8 encoding, https://www.fileformat.info/info/unicode/utf8.htm.

[22] Named Entity Recognition, https://en.wikipedia.org/wiki/Namedentity_recognition.

[23] spaCy NER training model, https://course.spacy.io/chapter4.

[24] Tiakas, E., Papadopoulos, A. N., Manolopoulos, Y., "Skyline queries: An introduction," In: $6^{\text {th }}$ International Conference on Information, Intelligence, Systems and Applications (IISA), DOI: 10.1109/IISA.2015.7388053, E-ISBN: 978-1-4673-9311-9, July (2015). 